\title{
INNOVATIONS IN CLINICAL PRACTICE
}

\section{PSST! I Need Help! Development of a Peer Support Program for Clinicians Having Serious Illness Conversations During COVID-19}

\author{
Jeffrey L. Greenwald, MD, SFHM ${ }^{\top}$ (D) Annah N. Abrams, MD², Elyse R. Park, PhD, MPH ${ }^{3}$, \\ Phuong L. Nguyen, $B A^{4}$, and Juliet Jacobsen, $M D^{4}$ \\ 'Department of Medicine, Massachusetts General Hospital, 50 Staniford Street, Suite 503B, Boston, MA, USA; ${ }^{2}$ Department of Child Psychiatry and \\ Pediatric Hematology Oncology, Massachusetts General Hospital, Boston, MA, USA; ${ }^{3}$ Departments of Psychiatry \& Medicine, Massachusetts \\ General Hospital, Boston, MA, USA; ${ }^{4}$ Continuum Project, Division of Palliative Care and Geriatrics, Massachusetts General Hospital, Boston, MA, USA.
}

J Gen Intern Med 36(4):1094-7

DOI: $10.1007 / \mathrm{s} 11606-020-06565-9$

(c) Society of General Internal Medicine 2021

\section{INTRODUCTION}

High-quality care involves balancing treatment options that are based on the best available evidence and clinical experience with care that aligns with patients' values and priorities. Despite the importance of understanding patients' values and priorities, conducting conversations with patients about them commonly meets with barriers that are patient-related (e.g., anxiety or denial), clinician-related (e.g., lack of time and training), and system-related (e.g., poor end-of-life systems and methods for documenting wishes). ${ }^{1}$ During times of crisis, such as the COVID-19 pandemic, these barriers may be amplified ${ }^{2-4}$ as patients face uncertainty and social isolation and clinicians face changing workflows, new clinical roles, and evolving diagnostic and prognostic information. ${ }^{5}$

Despite these barriers, the pandemic increases the importance of understanding patients' values and priorities. First, as is always true but especially so during times of stretched resources, it is important to avoid unwanted care. Second, insufficient anticipatory exploration of patients' priorities may result in rushed and poorly informed decisions should they become ill. $^{2}$

Prior to the pandemic, our hospital had trained over 1300 clinicians to conduct serious illness conversations (SIC) that explore patients' values and priorities using Ariadne Labs' Serious Illness Care Program. This program trained nonpalliative care providers how to conduct the conversation with the aid of a script. ${ }^{1,6}$ However, when the pandemic arose, our providers needed additional support. Since peer support for serious illness conversations has been shown to be valued by clinicians when it is accessible, tailored, and aligned with the clinician's goals, ${ }^{7}$ we developed the Peer SIC Support Team (PSST), a novel program to offer real-time assistance to

Received September 24, 2020

Accepted December 22, 2020

Published online January 26, 2021 frontline clinicians by leveraging trained, non-palliative care providers' communication skills.

\section{SETTING AND PARTICIPANTS}

The PSST was developed and implemented by the Continuum Project (www.mghcontinuumproject.org), the Serious Illness Care Program at the Massachusetts General Hospital, a large, academic affiliate of Harvard Medical School in Boston, MA. The service was available to clinicians in ambulatory and inpatient settings.

In March, 2020, we recruited and trained 44 volunteer peer supporters, including experienced members of the Continuum Project, members of our faculty coaching program from the hospital's Center for Physician Wellbeing, and members of our social work division. All of these individuals had undergone serious illness conversation training previously. Of note, very few of these individuals were specialists in palliative care but all were clinicians. These volunteers participated in two 1hour training sessions led by a palliative care attending. The training reviewed (1) the hospital's serious illness conversation guide (specifically modified for the pandemic; see Appendix) and (2) empathic language (scripted to assist clinicians in responding to patients' pandemic-related emotions and concerns developed by VitalTalk). ${ }^{8}$ In addition, peer supporters were familiarized with support materials which were compiled by or created for PSST and housed in a centralized webbased system, including tip sheets on how to document conversations and complete advance directives virtually.

Given the large number of volunteers recruited and the diverse support needs, the peer supporters were divided into two adult medical teams, a pediatric team, and a mental health team. The adult teams included adult primary care clinicians, social workers, and medical subspecialists. The pediatric team included adult and pediatric medical providers, social workers, and pediatric palliative care providers. The mental health team included psychologists, psychiatrists, and social workers available to assist clinicians using our service in need of more substantial or ongoing mental health care. Adult and pediatric 
peer supporters were able to refer these clinicians to the mental health team as needed.

\section{PROGRAM DESCRIPTION}

The PSST was designed with the following goals: (a) peer supporters will be a multidisciplinary group of individuals with communication training or experience; (b) reaching peer supporters when needed will be easy and fast; (c) peer supporters will have additional resources (personnel and webbased) to assist them; (d) there will be ongoing opportunities for the community of peer supporters to discuss their experiences with mental health support; and (e) the service will be confidential unless a peer supporter is concerned about a colleagues' safety.

\section{PSST ACTIVATION}

To request assistance from the PSST, a caller paged 4PSST (47778) through the hospital paging system. The PSST Lead, the individual carrying the PSST pager for the day, triaged the pages to the appropriate team. For example, a pediatric ICU resident's call would be triaged to the pediatric PSST team; an adult primary care provider call would be triaged to the adult team. The two adult teams alternated days of the week for responding to calls. The PSST service was available seven days a week from 7:00AM to 10:00PM. After hours, callers were directed to call back the next day or page palliative care for emergencies.

To route a caller to the appropriate team, the PSST established group texting through a mobile messaging application, WhatsApp, which all the peer supporters downloaded onto their cell phones. The Lead would send out a group text asking who was available to take the call since peer supporters had other responsibilities and did not have to be available all hours of the service. The larger group helped to ensure that someone was always available. When a peer supporter was identified, a private WhatsApp text was sent to this person, to preserve the anonymity of callers, with the details from the original page. The goal was to have the caller connect with a peer supporter within 10 minutes of placing the page. If no peer supporter was available to take the call within that timeframe, the Lead would take the call.

\section{CALL AND POST-CALL PROCEDURES}

The peer supporter's role was to support the caller's needs as well as to offer emotional support. This typically involved a combination of empathic listening, coaching callers around discreet aspects of communication, providing direct education around specific logistics (e.g., how to refer a patient to hospice or how to complete a health care proxy document remotely), and reinforcing the positive aspects of the caller's approach, while empathizing with how difficult and taxing these conversations often are and how normal it is to feel challenged by them. Peer supporters were advised against offering medical advice as this was not the purpose of the service. However, many peer supporters were physicians and felt comfortable, in certain circumstances, offering limited medical recommendations.

After the call, peer supporters were asked to share a brief, de-identified summary, the approximate length of the call, and any issues about which they needed additional guidance via the WhatsApp text group so others could learn from the call and share any advice or information requested. Peer supporters did not make notes in the charts of the patients discussed as the consult was for the clinician callers, not the patient.

\section{SUPPORT SESSIONS FOR PEER SUPPORTERS}

Each week, peer supporters were invited to join one of two optional, 1-hour video calls led by a psychiatrist and psychologist to discuss cases and receive feedback from the group leaders and peers. These sessions provided not only an opportunity for group learning but also a supportive peer environment.

\section{RESOURCES FOR PEER SUPPORTERS}

To ensure the peer supporters had access to up-to-date information regarding pandemic-related procedures, several resources were compiled and created (see Appendix). For example, the PSST had access to several web-based informational tip sheets that discussed logistical issues that commonly arise during clinicians' serious illness conversations with patients (e.g., how to complete a Massachusetts Order for Life-Sustaining Treatment (MOLST) form remotely, how to refer a patient to home-based palliative care, COVID-19 information).

Additionally, each WhatsApp text group included a few people who served as resources but did not take calls themselves. They included a chaplain, a psychiatrist, and members of the palliative care division. Their role was to monitor the texts in case a peer supporter posted a question to the group like "the caller has a patient who lives in [town]. What homebased palliative care programs serve that area?" Or "the caller says the patient is struggling with his relationship with his faith right now and I'm not sure how to respond." The resource members of the team could then offer guidance. Peer supporters were encouraged to refer callers to the Palliative Care Consult Service if needed.

\section{MARKETING AND DETAILING}

While the PSST was designed as a COVID-19-related program, we were open to any clinician needing guidance with serious illness conversations or related issues, irrespective of 
the patient's diagnosis. The PSST introduced and promoted its service through several mechanisms. First, we chose a name that would be memorable (as in, "Psst! I need some help!"). We sent targeted emails and had discussions with key nursing, physician, social work, and case management leaders of service in the ambulatory, ICU, and inpatient settings, and sent messages to residency directors and the MGH Center for Physician Wellbeing. We also sent hospital-wide informational emails. The PSST was presented at the hospital's Medical Grand Rounds and the Medicine COVID-19 Virtual Town Hall meetings. Additionally, members of the service conducted in-person detailing meetings with nursing leaders and other clinical staff on medical units and in the ICUs caring for COVID-19 patients and posted flyers there. Peer supporters were also encouraged to present the service to their colleagues.

\section{COST}

The primary expense for this service was administrative time. A project manager oversaw many of the PSST logistics (scheduling trainings and peer support groups, ensuring the Lead pager was covered, compiling and posting resources, setting up and tracking the WhatsApp text groups). The upfront time commitment to establish the PSST was moderate (approximately 10 hours/week) and lessened once it was underway (approximately 4 hours/week). The peer supporters were volunteers. The expense for the trainer and oversite of the program by the director of the PSST were subsumed under the existing hospital's Serious Illness Care Program. WhatsApp is a free app and none of the additional personnel resources or printed resources costs money to use.

\section{PROGRAM EVALUATION}

PSST was evaluated on multiple levels. First, service utilization was tracked through the number of callers each day posted to the WhatsApp groups. From the start of the service on March 31, 2020, until the peak of the pandemic had passed for our hospital in late April, we received an average of 1.1 calls/day (range $0-4$ calls daily with $72 \%$ taken by peer supporters and $28 \%$ by Leads). Thereafter, calls slowed markedly, with 45 calls in total by May 11, 2020, after which no further calls were received and we closed the service July 3, 2020. All but one call was triaged to an adult PSST group and the other to the pediatric team. The calls were taken by 17 individual team members. Call duration was also tracked with calls averaging 13 minutes (range 3.5-30; standard deviation 6.5 minutes).

Additionally, we collected themes of calls (see Table 1). This information was used to promote discussions during the support sessions for peer supporters and identify needed resources. After the pandemic's peak and call volume dropped, a peer supporter survey was conducted to assess their PSST experience. It demonstrated that peer supporters who
Table 1 PSST Call Themes

\begin{tabular}{l}
\hline 1. Documentation questions \\
a. Serious illness conversations \\
b. Change in code status \\
c. Completing a MOLST remotely \\
2. Care guidance \\
a. Hospice resources \\
b. Available resources for patients who do not want to come to the \\
emergency department \\
c. How to have serious illness conversations \\
d. Help with talking to health care proxies \\
3. Team distress \\
a. Wanting to honor family wishes but not being allowed to have \\
visitors \\
b. Patients dying alone \\
c. Help with debriefing after a distressing COVID-19 deaths \\
d. Wanting to honor patient's wishes (help with honoring patient \\
autonomy vs. desire to do no harm) \\
4. Hospital health care providers \\
a. Supporting new roles (e.g., outpatient providers working as \\
hospitalists) \\
b. Talking through difficult conversations and follow-up calls after \\
difficult conversations \\
5. Medical care questions \\
a. Symptom management \\
b. Redirecting calls to palliative care
\end{tabular}

MOLST, medical order for life-sustaining treatment

had conducted at least one call were more likely to agree or strongly agree that their participation on PSST was meaningful to them compared with those who had not conducted a call $(100 \%$ vs. $55 \%)$. None of the survey respondents felt that the time commitment was "too much."

\section{DISCUSSION}

Clarifying patients' values and priorities is always important but especially so during a pandemic when many people will rapidly become seriously ill. Herein, we describe the design and implementation of a novel service, the PSST, which utilized non-palliative care, volunteer peer supporters who offered rapid, confidential assistance to clinicians working to clarify the values and priorities of their patients across settings from ambulatory care to the ICUs. This service required moderate, upfront, administrative time but was otherwise a low-cost program. It gave an opportunity for clinicians with advanced communication skills to build a community through which they could offer important support to frontline clinicians which the peer supporters found rewarding. PSST took place in a hospital with an established program in serious illness conversation training and leveraged its staff with skills in this space, which may limit its replicability in other settings. However, serious illness conversation training materials are open access ${ }^{6}$ and many hospitals have access to social workers and others with interests and skills in communication who could participate in such a service. Thus, this service could be replicated in other institutions for this or future crises.

Corresponding Author: Jeffrey L. Greenwald, MD, SFHM; Department of Medicine, Massachusetts General Hospital, 50 Staniford Street, Suite 503B, Boston, MA 02114, USA (e-mail: jlgreenwald@partners.org). 
Supplementary Information The online version contains supple mentary material available at https://doi.org/10.1007/s11606-02006565-9.

\section{Compliance with Ethical Standards:}

Conflict of Interest: None of the authors report a conflict of interest with the work presented herein.

\section{REFERENCES}

1. Bernacki RE. Block SD, and the American College of Physicians High Value Care Task, Communication about serious illness care goals: a review and synthesis of best practices. JAMA Intern Med 2014;174(12):19942003.

2. Curtis JR, Kross EK, Stapleton RD. Care planning and decisions about do-not-resuscitate orders during Novel Coronavirus 2019 (COVID-19). JAMA 2020;323(18):1771-2.
3. Rosenberg LB, Greenwald JL, Caponi B, et al. Confidence with and barriers to serious illness communication: a national survey of hospitalists. J Pall Med 2017;20(9): 1013-9.

4. Taylor S, Landry CA, Paluszek MM, et al. Development and initial validation of the COVID Stress Scales. J Anx Disorders. 2020;72. Available online at: https://doi.org/10.1016/j.janxdis.2020.102232. Accessed: 8.26.20.

5. Ehrlich H, McKenney M, Elkbuli A. Protecting our healthcare workers during the COVID-19 pandemic. Am J Emerg Med 2020;38(7):1527-8.

6. Serious Illness Care. Ariadne Labs. Boston, Massachusetts. Available online at: https://www.ariadnelabs.org/areas-of-work/serious-illnesscare/. Accessed: 11.4.20.

7. Jacobsen J, Cole CA, Daubman BR, et al. A novel use of peer coaching to teach primary palliative care skills: coaching consultation. J Pain Symptom Manage 2017;54(4):578-582.

8. VitalTalk COVID Resources. Available online at: https://www.vitaltalk.org/ covid-resources. Accessed: 11.4.20.

Publisher's Note Springer Nature remains neutral with regard to jurisdictional claims in published maps and institutional affiliations. 